they could test tuning for different facial characteristics $^{4,5,7}$. Face cells are rare, when one must stumble randomly through the temporal lobe looking for them.

This is not merely a technical weakness, as experiments almost always require searching for the relevant cells using the same stimulus set that will be ultimately used to test selectivity for features. Thus, the selection procedure can end up finding not the general properties of face cells, but face cells whose variance is likely to be explained by the feature variation contained in the stimulus set. Identifying the face patches independently, using faces in an independent fMRI experiment, as was done here, avoids this particular selection bias. Furthermore, the high likelihood of finding face selective cells in the patch means that tuning variations can be more systematically tested after finding the relevant cell. The greater yield (a technical advance) means that more hypotheses about tuning can be tested. Finally, because cells in the patch share characteristics, the likelihood that the sampled cells represent common properties of the local circuitry is increased.

At the same time, these results also conflict with other studies. For example, one study found that face familiarity significantly modulates the responses of superior temporal sulcus face cells ${ }^{5}$. Another ${ }^{8}$ found that many face cells responded best to the average rather than to extreme values. These differences could potentially result from these studies not looking at the same temporal lobe face patch. This is an important caveat; the scheme here may not hold in other face patches. In other face-selective areas, learning $5,7,9$, adaptation or norming ${ }^{8}$, or hypothesis testing ${ }^{10}$ might be more important. This study ${ }^{6}$ offers hope for examining those cells as well, as fMRI identifies multiple face patches in the temporal lobe, potentially allowing each (and the interactions among them) to be examined in turn.

Cells in the face patches identified here are selective for a small number of features in a grandmother's face, in the context of a larger face, and they are tuned to specific dimensions of this feature. These results vindicate some theories of visual object selectivity ${ }^{11}$. In addition, although the identification of a homogenous region of face cells in this study allowed for the testing of a large stimulus set, face space could still be more densely and systematically explored by recursive algorithms that explore relevant spaces $^{12}$. Other questions also remain. Will these features be just as effective at driving cells in different face contexts, for example, in face composites? Will features identified in cartoon faces produce similar modulation in real faces? How do these cells respond to ambiguous $^{9}$, imagined ${ }^{13}$ or learned $d^{5,7,9}$ faces? Will learning alter the feature dependence of responses ${ }^{8,11}$. It remains to be seen whether the coding described here still applies in these more complex situations.

1. Svoboda, E. Faces, faces, everywhere. New York Times, 13 February 2007.

2. Quiroga, R.Q., Reddy, L., Kreiman, G., Koch, C. \& Fried, I. Nature 435, 1102-1107 (2005).

3. Desimone, R., Albright, T.D., Gross, C.G. \& Bruce, C J. Neurosci. 4, 2051-2062 (1984).

4. Yamane, S., Kaji, S. \& Kawano, K. Exp. Brain Res. 73 209-214 (1988)

5. Young, M.P. \& Yamane, S. Science 256, 1327-1331 (1992).

6. Freiwald, W.A., Tsao, D.Y. \& Livingstone, M.S. Nat Neurosci. 12, 1187-1196 (2009)

7. Sigala, N. \& Logothetis, N.K. Nature $415,318-320$ (2002).

8. Leopold, D.A., Bondar, I.V. \& Giese, M.A. Nature 442 , 572-575 (2006)

9. Tovee, M.J., Rolls, E.T. \& Ramachandran, V.S Neuroreport 7, 2757-2760 (1996).

10. Bar, M. et al. Proc. Natl. Acad. Sci. USA 103, 449454 (2006).

11. Baker, C.I., Behrmann, M. \& Olson, C.R. Nat. Neurosci. 5, 1210-1216 (2002).

12. Yamane, Y., Carlson, E.T., Bowman, K.C., Wang, Z. \& Connor, C.E. Nat. Neurosci. 11, 1352-1360 (2008).

13. Kreiman, G., Koch, C. \& Fried, I. Nature 408, 357-361 (2000).

\title{
Reconnecting injured nerves
}

Severed axonal connections do not spontaneously regenerate, creating a major hurdle for functional recovery following spinal cord injury (SCI). Previous attempts to aid axonal regeneration have failed to show correct reinnervation of specific target sites in the brain. On pp. 1106-1113 of this issue, Alto et al. demonstrate the successful anatomical regrowth of rat hind limb sensory nerve into the brainstem, across the SCI site.

The Tuszynski laboratory has previously shown that a combination of local neurotrophin expression, conditioning peripheral nerve injury, and cell grafts applied to and near a lesion site can provoke the partial regrowth of severed sensory axons after SCI. In these experiments, the lentiviral expression of neurotrophin-3 (NT-3) provided the chemoattractive guidance cue to direct regenerating axons. A bone marrow graft at the injury site provided scaffolding, a kind of cellular bridge, for the regenerating nerve tract. Sciatic nerve preconditioning lesions, a manipulation known to promote axonal regeneration, were also used. The regenerating axons grew into and beyond the spinal cord lesion site, but the regenerated axons in these previous studies did not quite reach their original targets in the brainstem.

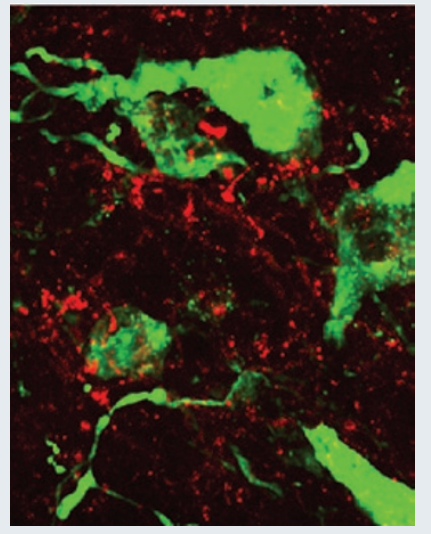

Alto et al. now report ascending sensory tracts that successfully reconnect with their correct target site, the nucleus gracilis in the brain stem. To achieve this, the authors moved the injury site to level C1 on the spinal cord (compared with C4 in their earlier work), where the distance to the target site is much shorter $(\sim 2 \mathrm{~mm})$. The picture shows a confocal micrograph of transganglionic labeling of injured sensory axons (cholera toxin B subunit labeling in red) reaching retrogradely labeled target neurons in the nucleus gracilis (Fluorogold labeling in green). To demonstrate the importance of local neurotrophin gradients in coaxing the regenerating axon to the correct site, the authors misexpressed NT-3 at an inappropriate location, the medullary reticular formation, and found regenerating axons being misdirected and inappropriately reconnected.

Disappointingly, despite this successful anatomical reconnection, these rats did not show appreciable functional recovery. Although regenerating axons formed ultrastructures that are consistent with de novo synaptic contacts, the target neurons in the brain stem showed little or no response to electrical stimulation of the sciatic nerve. Despite the lack of functional recovery, this study shows that proper chemotrophic guidance is a crucial step in promoting recovery after SCI. These findings can potentially aid in designing a combinatorial therapeutic strategy for individuals who have lost peripheral functions as a result of spinal cord trauma.

Min Cho 